\title{
Theory of rubber friction: Nonstationary sliding
}

\author{
B. N. J. Persson and A. I. Volokitin \\ IFF, FZ-Jülich, 52425 Jülich, Germany \\ (Received 31 May 2001; published 19 March 2002)
}

\begin{abstract}
When rubber slides on a hard, rough substrate, the surface asperities of the substrate exert oscillating forces on the rubber surface leading to energy "dissipation" via the internal friction of the rubber. In this paper we extend an earlier published theory [B.N.J. Persson, J. Chem. Phys. 115, 3840 (2001)] to nonstationary sliding, and present a discussion of how the area of real contact and the friction force depend on the nature of the substrate surface roughness and on the history of the sliding motion. We consider in detail the case when the substrate surface has a self-affine fractal structure.
\end{abstract}

DOI: 10.1103/PhysRevB.65.134106

PACS number(s): 81.40.Pq, 62.20.-x

\section{INTRODUCTION}

The nature of the friction when rubber slides on a hard substrate is a topic of considerable practical importance, e.g., for the construction of tires, ${ }^{1}$ wiper blades, ${ }^{1}$ and in the cosmetics industry. ${ }^{2}$ Rubber friction differs in many ways from the frictional properties of most other solids. The reason for this is the very low elastic modulus of rubber and the high internal friction exhibited by rubber over a wide frequency region.

The pioneering studies of Grosch ${ }^{3}$ have shown that rubber friction in many cases is directly related to the internal friction of the rubber. Thus experiments with rubber surfaces sliding on silicon carbide paper and glass surfaces give friction coefficients with the same temperature dependence as that of the complex elastic modulus $E(\omega)$ of the rubber. In particular, there is a marked change in friction at high speeds and low temperatures, where the rubber's response is driven into the so-called glassy region. In this region, the friction shows marked stick slip and falls to a level of $\mu \approx 0.4$, which is more characteristic of plastics. This proves that the friction force under most normal circumstances is directly related to the internal friction of the rubber, i.e., it is mainly a bulk property of the rubber. ${ }^{3}$

Almost all real surfaces have roughness on many different length scales which must be taken into account when calculating the rubber sliding friction force. This was considered in the work by Klüppel and Heinrich. ${ }^{4}$ However, in Ref. 4 the deformation of the rubber surface in response to the surface roughness is only included in some average way. One of us has developed a theory of rubber friction where the deformations of the rubber are taken into account on all relevant length scales. ${ }^{5}$ The theories in Refs. 4 and 5 consider rubber friction when a rubber block is slid at constant velocity over a hard, rough surface. Other studies of this topic are presented in Refs. 1 and 6-10. In this paper we extend the theory in Ref. 5 to nonstationary sliding. The theory is valid for arbitrary (random) surface roughness, but explicit results are presented for self-affine fractal surface profiles. ${ }^{11,12}$ Such surfaces "look the same" when magnified by a scaling factor $\zeta$ in the $x y$ plane of the surface and by a factor $\zeta^{H}$ (where $0<H<1$ ) in the perpendicular $z$ direction. We note that many materials of practical importance have (approximately) self-affine fractal surfaces. Thus, for example, road surfaces and the surfaces of many cleaved, brittle materials tend to be self-affine fractal with the fractal dimension $D_{f}=3-H$ $\approx 2.2-2.5$. In practice there is always a lower, $\lambda_{1}$, and upper, $\lambda_{0}$, cutoff length, so that the surface is self-affine fractal only when viewed in a finite length-scale interval $\lambda_{1}<\lambda$ $<\lambda_{0}$. For surfaces produced by brittle fracture, the upper cutoff length $\lambda_{0}$ is usually identical to the lateral size $L$ of the fracture surface. This may also be the case for many surfaces of engineering importance (see, e.g., Refs. 13-17). However, for road surfaces the upper cutoff $\lambda_{0}$ is of the order of a few millimeters, which corresponds to the size of the largest sand particles in the asphalt. Less is known about the shortdistance cutoff $\lambda_{1}$, but below, it will be assumed to be of the order of a few micrometers, so that the length-scale region where the road surface may be assumed to be fractal may extend over $\sim$ three orders of magnitude.

In spite of its great practical importance, very few detailed experimental studies of the nonstationary frictional dynamics of rubber on a hard rough substrate have been performed. Recently Ronsin and Coeyrehourcq ${ }^{18}$ have studied (experimentally) the state-, rate-, and temperature-dependent friction of elastomer, but focused mainly on the glassy side of the dynamical response of the polymer. An early study by Roberts and Thomas focused on some simple nonstationary sliding problems involving rubber. ${ }^{19}$

When rubber slides on a hard, rough surface with roughness on the length scales $\lambda$, it will be exposed to fluctuating forces with frequencies $\omega \sim v / \lambda$. Since we have a wide distribution of length scales $\lambda_{1}<\lambda<\lambda_{0}$, we will have a corresponding wide distribution of frequency components in the Fourier decomposition of the surface stresses acting on the sliding rubber block. The contribution to the friction coefficient $\mu$ from surface roughness on the length scale $\lambda$ will be maximal when $v / \lambda \approx 1 / \tau$, where $1 / \tau$ is the frequency when $\operatorname{Im} E(\omega) /|E(\omega)|$ is maximal, which is located in the transition region between the rubbery region (low frequencies) and the glassy region (high frequencies). We can interpret $1 / \tau$ as a characteristic rate of flips of molecular segments (configurational changes), which are responsible for the viscoelastic properties of the rubber. Since the flipping is a thermally activated process it follows that $\tau$ depends exponentially (or faster) on the temperature $\tau \sim \exp \left(\Delta E / k_{B} T\right)$, where $\Delta E$ is the barrier involved in the transition. In reality, there is a 
wide distribution of barrier heights $\Delta E$ and hence of relaxation times $\tau$, and the transition from the rubbery region to the glassy region is very wide, typically extending over three orders of magnitude in frequency.

\section{SLIDING FRICTION}

This section is based on the formalism developed in Ref. 5. Using the theory of elasticity (assuming an isotropic elastic medium for simplicity), one can calculate the displacement field $u_{i}$ on the surface $z=0$ in response to the surface stress distributions $\sigma_{i}=\sigma_{3 i}$. Let us define the Fourier transforms

$$
\begin{gathered}
u_{i}(\mathbf{q}, \omega)=\frac{1}{(2 \pi)^{3}} \int d^{2} x d t u_{i}(\mathbf{x}, t) e^{-i(\mathbf{q} \cdot \mathbf{x}-\omega t)}, \\
u_{i}(\mathbf{x}, t)=\int d^{2} q d \omega u_{i}(\mathbf{q}, \omega) e^{i(\mathbf{q} \cdot \mathbf{x}-\omega t)},
\end{gathered}
$$

and similar for $\sigma_{i}(\mathbf{q}, \omega)$. Here $\mathbf{x}=(x, y)$ and $\mathbf{q}=\left(q_{x}, q_{y}\right)$ are two-dimensional vectors. We have ${ }^{5}$

$$
u_{i}(\mathbf{q}, \omega)=M_{i j}(\mathbf{q}, \omega) \sigma_{j}(\mathbf{q}, \omega)
$$

or, in matrix form,

$$
\mathbf{u}(\mathbf{q}, \omega)=M(\mathbf{q}, \omega) \boldsymbol{\sigma}(\mathbf{q}, \omega),
$$

where the matrix $M$ is given in Ref. 5 .

We now assume that the surface stress $\sigma(\mathbf{q}, \omega)$ only acts in the $z$ direction (see below) so that

$$
u_{z}(\mathbf{q}, \omega)=M_{z z}(\mathbf{q}, \omega) \boldsymbol{\sigma}_{z}(\mathbf{q}, \omega) .
$$

Since $\omega \sim v q$ (where $v$ is a typical sliding velocity) we get $\omega / c_{T} q \sim v / c_{T} \ll 1$ in most cases of practical interest. Thus, we can expand $M(q, \omega)$ to leading order in $\omega / c_{T} q$. This gives $^{5}$

$$
\left(M_{z z}\right)^{-1}=-\frac{E q}{2\left(1-\nu^{2}\right)} .
$$

It is interesting to note that if instead of assuming that the surface stress acts in the $z$ direction we assume that the displacement $\mathbf{u}$ points along the $z$ direction, then

$$
\sigma_{z}(\mathbf{q}, \omega)=\left(M^{-1}\right)_{z z}(\mathbf{q}, \omega) u_{z}(\mathbf{q}, \omega),
$$

where in the limit $\omega / c_{T} q \ll 1$,

$$
\left(M^{-1}\right)_{z z}=-\frac{2 E q(1-\nu)}{(1+\nu)(3-4 \nu)},
$$

which differs from Eq. (2) only with respect to a factor $4(1-\nu)^{2} /(3-4 \nu)$. For rubberlike materials $(\nu \approx 0.5)$ this factor is of order unity. Hence, practically identical results are obtained independently of whether one assumes that the interfacial stress or displacement vector are perpendicular to the nominal contact surface. In reality, neither of these two assumptions holds strictly, but the result above indicates that the theory is not sensitive to this approximation.
We now make the basic assumption that during sliding the whole rubber interfacial surface area moves forwards according to $-\mathbf{r}(t)$, i.e., we assume that no Schallamach wave propagation or local interfacial stick-slip motion (where different interfacial areas slip at different times) occurs (see Ref. 20). At this point it is convenient to introduce a coordinate system with the $x y$ plane fixed in the (undeformed) bottom surface of the rubber block, and consider the substrate as moving with the velocity $\dot{\mathbf{r}}(t)$. We first assume that the rubber block is in complete contact with the substrate during sliding. Thus, the rubber block surface displacement field induced by the substrate roughness is

$$
\mathbf{u}(\mathbf{x}, t)=\mathbf{u}[\mathbf{x}-\mathbf{r}(t)],
$$

where $\mathbf{r}(t)=x(t) \hat{x}$ and

$$
\begin{aligned}
\mathbf{u}(\mathbf{q}, \omega) & =\frac{1}{(2 \pi)^{3}} \int d^{2} x d t \mathbf{u}[\mathbf{x}-\mathbf{r}(t)] e^{-i(\mathbf{q} \cdot \mathbf{x}-\omega t)} \\
& =\frac{1}{(2 \pi)^{3}} \int d^{2} x d t \mathbf{u}(\mathbf{x}) e^{-i\{\mathbf{q} \cdot[\mathbf{x}+\mathbf{r}(t)]-\omega t\}} \\
& =\mathbf{u}(\mathbf{q}) f(\mathbf{q}, \omega)
\end{aligned}
$$

where

$$
\mathbf{u}(\mathbf{q})=\frac{1}{(2 \pi)^{2}} \int d^{2} x \mathbf{u}(\mathbf{x}) e^{-i \mathbf{q} \cdot \mathbf{x}}
$$

and

$$
f(\mathbf{q}, \omega)=\frac{1}{2 \pi} \int d t e^{-i[\mathbf{q} \cdot \mathbf{r}(t)-\omega t]} .
$$

If $\sigma_{f}(t)$ denotes the frictional shear stress acting on the bottom surface of the rubber block, then the instantaneous power absorption $P(t)=\sigma_{f}(t) A_{0} \dot{x}(t)$ must be given by the rate of work by the substrate surface asperities on the rubber block:

$$
\sigma_{f}(t) A_{0} \dot{x}(t)=\int d^{2} x\langle\dot{\mathbf{u}}(\mathbf{x}, t) \cdot \sigma(\mathbf{x}, t)\rangle
$$

where the terms inside the angular brackets $\langle\cdots\rangle$ denotes the ensemble average, and where $A_{0}$ is the surface area. But

$$
\begin{aligned}
\dot{\mathbf{u}}(\mathbf{x}, t)=\dot{\mathbf{u}}[\mathbf{x}-\mathbf{r}(t)] & =-\dot{\mathbf{r}}(t) \cdot \nabla \mathbf{u}[\mathbf{x}-\mathbf{r}(t)] \\
& =-\dot{x}(t) \frac{\partial}{\partial x} \mathbf{u}[\mathbf{x}-\mathbf{r}(t)] .
\end{aligned}
$$

Thus, using Eqs. (5) and (6),

$$
\sigma_{f}(t)=-\frac{1}{A_{0}} \int d^{2} x\left\langle\frac{\partial}{\partial x} \mathbf{u}(\mathbf{x}, t) \cdot \sigma(\mathbf{x}, t)\right\rangle .
$$

Using Eqs. (1) and (7) gives 


$$
\begin{aligned}
\sigma_{f}(t)= & \frac{(2 \pi)^{2}}{A_{0}} \int d^{2} q d \omega d \omega^{\prime}\left(-i q_{x}\right) e^{-i\left(\omega+\omega^{\prime}\right) t} \\
& \times\left\langle\mathbf{u}(\mathbf{q}, \omega) \cdot \boldsymbol{\sigma}\left(-\mathbf{q},-\omega^{\prime}\right)\right\rangle \\
\approx & \frac{(2 \pi)^{2}}{A_{0}} \int d^{2} q d \omega d \omega^{\prime}\left(-i q_{x}\right) e^{-i\left(\omega+\omega^{\prime}\right) t} \\
& \times\left(M_{z z}\right)^{-1}\left(-\mathbf{q}, \omega^{\prime}\right)\left\langle u_{z}(\mathbf{q}, \omega) u_{z}\left(-\mathbf{q},-\omega^{\prime}\right)\right\rangle .
\end{aligned}
$$

Substituting Eqs. (3) in (8) gives

$$
\begin{aligned}
\sigma_{f}(t)= & \frac{(2 \pi)^{2}}{A_{0}} \int d^{2} q d \omega d \omega^{\prime}\left(-i q_{x}\right) e^{-i\left(\omega+\omega^{\prime}\right) t} \\
& \times\left(M_{z z}\right)^{-1}\left(-\mathbf{q}, \omega^{\prime}\right) f(\mathbf{q}, \omega) f\left(-\mathbf{q}, \omega^{\prime}\right)\left\langle u_{z}(\mathbf{q}) u_{z}(-\mathbf{q})\right\rangle .
\end{aligned}
$$

Let us now consider sliding on a randomly rough surface described by the function $z=h(\mathbf{x})$ [where $\mathbf{x}=(x, y)]$. Assume first that the rubber is able to deform and completely follow the substrate surface profile and that $|\nabla h(\mathbf{x})| \ll 1$. Thus we can approximate $u_{z} \approx h(\mathbf{x})$. Using Eq. (9) gives

$$
\begin{aligned}
\sigma_{f}(t)= & \frac{(2 \pi)^{2}}{A_{0}} \int d^{2} q d \omega d \omega^{\prime}\left(-i q_{x}\right) e^{-i\left(\omega+\omega^{\prime}\right) t} \\
& \times\left(M_{z z}\right)^{-1}\left(-\mathbf{q}, \omega^{\prime}\right) f(\mathbf{q}, \omega) f\left(-\mathbf{q}, \omega^{\prime}\right)\langle h(\mathbf{q}) h(-\mathbf{q})\rangle,
\end{aligned}
$$

where we assumed that $\langle h\rangle=0$. Now, note that

$$
\begin{aligned}
\langle h(\mathbf{q}) h(-\mathbf{q})\rangle & =\frac{A_{0}}{(2 \pi)^{4}} \int d^{2} x\langle h(\mathbf{x}) h(\mathbf{0})\rangle e^{-i \mathbf{q} \cdot \mathbf{x}} \\
& \equiv \frac{A_{0}}{(2 \pi)^{2}} C(q),
\end{aligned}
$$

since $\left\langle h(\mathbf{x}) h\left(\mathbf{x}^{\prime}\right)\right\rangle$ depends only on the difference $\mathbf{x}-\mathbf{x}^{\prime}$. The spectral density $C(q)$ is defined by

$$
C(q)=\frac{1}{(2 \pi)^{2}} \int d^{2} x\langle h(\mathbf{x}) h(\mathbf{0})\rangle e^{-i \mathbf{q} \cdot \mathbf{x}} .
$$

Substituting Eqs. (11) in (10) and using Eq. (2) gives

$$
\begin{aligned}
\sigma_{f}(t)= & \int d^{2} q q^{2} \cos \phi C(q) \int d \omega d \omega^{\prime} e^{-i\left(\omega+\omega^{\prime}\right) t} f(\mathbf{q}, \omega) \\
& \times f\left(-\mathbf{q}, \omega^{\prime}\right) \frac{i E\left(\omega^{\prime}\right)}{2\left(1-\nu^{2}\right)}
\end{aligned}
$$

where we have used polar coordinates so that $q_{x}=q \cos \phi$. Now, note that

$$
\int d \omega f(\mathbf{q}, \omega) e^{-i \omega t}=f(\mathbf{q}, t)=e^{-i \mathbf{q} \cdot \mathbf{r}(t)}
$$

and

$$
\begin{gathered}
\int d \omega^{\prime} f\left(-\mathbf{q}, \omega^{\prime}\right) \frac{E\left(\omega^{\prime}\right)}{2\left(1-\nu^{2}\right)} e^{-i \omega^{\prime} t} \\
=\frac{1}{2 \pi} \int d t^{\prime} e^{i \mathbf{q} \cdot \mathbf{r}\left(t^{\prime}\right)} \frac{E\left(t-t^{\prime}\right)}{2\left(1-\nu^{2}\right)}
\end{gathered}
$$

Using these equations, Eq. (13) gives

$$
\begin{aligned}
\sigma_{f}(t)= & \frac{1}{2 \pi} \int d^{2} q q^{2} \cos \phi C(q) \\
& \times \int d t^{\prime} e^{-i \mathbf{q} \cdot\left[\mathbf{r}(t)-\mathbf{r}\left(t^{\prime}\right)\right]} \frac{i E\left(t-t^{\prime}\right)}{2\left(1-\nu^{2}\right)} .
\end{aligned}
$$

If we define

$$
F(\mathbf{q}, t)=-\frac{1}{2 \pi} \int d t^{\prime} \frac{E\left(t-t^{\prime}\right)}{\sigma_{0}\left(1-\nu^{2}\right)} e^{-i \mathbf{q} \cdot\left[\mathbf{r}(t)-\mathbf{r}\left(t^{\prime}\right)\right]},
$$

we get

$$
\sigma_{f}(t)=\frac{1}{2} \sigma_{0} \int d^{2} q q^{2} \cos \phi C(q) \operatorname{Im} F(\mathbf{q}, t)
$$

The friction coefficient $\mu$ can be obtained by dividing the frictional shear stress (18) with the pressure $\sigma_{0}$ :

$$
\mu(t)=\frac{1}{2} \int d^{2} q q^{2} \cos \phi C(q) P(q, t) \operatorname{Im} F(\mathbf{q}, t) .
$$

In Eq. (19) we have introduced an additional factor $P(q, t)$, defined as the fraction of the original macrocontact area where contact remains when we study the contact area on the length scale $\lambda=2 \pi / q$ (see below). In principle, $\nu$ depends on frequency but the factor $1 /\left(1-\nu^{2}\right)$ varies from $4 / 3$ $\approx 1.33$ for $\nu=0.5$ (rubbery region) to $\approx 1.19$ for $\nu=0.4$ (glassy region) and we can neglect the weak dependence on frequency.

Since $C(q)$ and $P(q, t)$ only depend on the magnitude of q, from Eq. (19),

$$
\mu(t)=\frac{1}{2} \int d q q^{3} C(q) P(q, t) \int d \phi \cos \phi \operatorname{Im} F(\mathbf{q}, t) .
$$

Note that the factor $\cos \phi$ in the integrand vanishes when $\phi=\pi / 2$, while it is maximal when $\phi=0$. This has a simple but important physical origin: Consider two cosine-surface corrugations, where the "wave vector" points (i) along the $x$ axis (the sliding direction), and (ii) along the $y$ axis. The former case corresponds to $\phi=0$, and in this case the rubber block will experience pulsating deformations during sliding along the $x$ axis. The second case corresponds to $\phi=\pi / 2$, where the elastic deformations of the rubber do not change during sliding along the $x$ axis, and this type of surface roughness will therefore not contribute to the friction. The 
function $P(q, t)$ was derived in Ref. 5 for the case of stationary sliding, and the same derivation is valid in the present case. Thus,

$$
P(q, t)=\frac{2}{\pi} \int_{0}^{\infty} d x \frac{\sin x}{x} \exp \left[-x^{2} G(q, t)\right],
$$

where

$$
G(q, t)=\frac{\left\langle\sigma_{z}^{2}(t)\right\rangle_{q}}{2 \sigma_{0}^{2}} .
$$

Here $\left\langle\sigma_{z}^{2}(t)\right\rangle_{q}$ is the average of the square of the interfacial stress, where the average only includes the roughness wave vectors with magnitude smaller than $q$, and where it is assumed that contact with the substrate occurs everywhere. Now, note that

$$
\begin{aligned}
\left\langle\sigma_{z}^{2}(t)\right\rangle & =\frac{(2 \pi)^{2}}{A_{0}} \int d^{2} q d \omega d \omega^{\prime} e^{-i\left(\omega+\omega^{\prime}\right) t}\left\langle\sigma_{z}(\mathbf{q}, \omega) \sigma_{z}\left(-\mathbf{q}, \omega^{\prime}\right)\right\rangle \\
& =\int d^{2} q d \omega d \omega^{\prime} e^{-i\left(\omega+\omega^{\prime}\right) t} M_{z z}^{-1}(\mathbf{q}, \omega) M_{z z}^{-1}\left(-\mathbf{q}, \omega^{\prime}\right) C(q) f(\mathbf{q}, \omega) f\left(-\mathbf{q}, \omega^{\prime}\right) \\
& =\frac{1}{4} \int d^{2} q d \omega d \omega^{\prime} q^{2} e^{-i\left(\omega+\omega^{\prime}\right) t} \frac{E(\omega)}{1-\nu^{2}} \frac{E\left(\omega^{\prime}\right)}{1-\nu^{2}} C(q) f(\mathbf{q}, \omega) f\left(-\mathbf{q}, \omega^{\prime}\right) \\
& =\frac{1}{4} \int d^{2} q q^{2} C(q) \mid \frac{1}{2 \pi} \int d t^{\prime} \frac{E\left(t-t^{\prime}\right)}{1-\nu^{2}} e^{\left.i \mathbf{q} \cdot \mathbf{r}\left(t^{\prime}\right)\right|^{2}} \\
& =\frac{1}{4} \sigma_{0}^{2} \int d q q^{3} C(q) \int d \phi|F(\mathbf{q}, t)|^{2} .
\end{aligned}
$$

Thus

$$
G(q, t)=\frac{1}{8} \int_{q_{L}}^{q} d q q^{3} C(q) \int d \phi|F(\mathbf{q}, t)|^{2} .
$$

Let us summarize the basic results obtained above. The friction coefficient $\mu(t)$ for a flat rubber surface sliding on a nominally flat substrate in the most general case is given by

$$
\mu(t)=\frac{1}{2} \int d q q^{3} C(q) P(q, t) \int d \phi \cos \phi \operatorname{Im} F(\mathbf{q}, t),
$$

where the (normalized) area of contact $A(\lambda) / A_{0}$, on the length scale $\lambda=2 \pi / q$, is given by

$$
\begin{aligned}
P(q, t) & =\frac{2}{\pi} \int_{0}^{\infty} d x \frac{\sin x}{x} \exp \left[-x^{2} G(q, t)\right], \\
G(q, t) & =\frac{1}{8} \int_{q_{L}}^{q} d q q^{3} C(q) \int d \phi|F(\mathbf{q}, t)|^{2} .
\end{aligned}
$$

In the equations above

$$
F(\mathbf{q}, t)=-\frac{1}{2 \pi} \int d t^{\prime} \frac{E\left(t-t^{\prime}\right)}{\sigma_{0}\left(1-\nu^{2}\right)} e^{-i \mathbf{q} \cdot\left[\mathbf{r}(t)-\mathbf{r}\left(t^{\prime}\right)\right]} .
$$

Note that if $E(t)$ changes slowly with $t$, then in Eq. (28) we can expand

$$
\mathbf{r}\left(t^{\prime}\right) \approx \mathbf{r}(t)+\dot{\mathbf{r}}(t)\left(t^{\prime}-t\right)
$$

so that

$$
F(\mathbf{q}, t)=-\frac{1}{2 \pi} \int d t^{\prime} \frac{E\left(t-t^{\prime}\right)}{\sigma_{0}\left(1-\nu^{2}\right)} e^{-i \mathbf{q} \cdot \dot{\mathbf{r}}(t)\left(t-t^{\prime}\right)} .
$$

Substituting this in Eqs. (25)-(27) gives $\mu(t)=\mu_{k}[\dot{x}(t)]$, i.e., the friction coefficient depends only on the instantaneous sliding velocity. The necessary condition for the validity of the expansion (29) in Eq. (28) is that

$$
q|\ddot{x}(t)|\left(\tau^{*}\right)^{2} \ll 1
$$

where $\tau^{*}$ is the memory time of the kernel $E(t)$ [see Eq. (38a)].

We consider now the limit $\sigma_{0} \ll E(\omega=0)$, which is satisfied in most applications. In this case, for most $q$ values of interest, $G(q, t) \gg 1$, so that only $x \ll 1$ will contribute to the integral in Eq. (26), we can approximate $\sin x \approx x$, and

$$
P(q, t) \approx \frac{2}{\pi} \int_{0}^{\infty} d x \exp \left[-x^{2} G(q, t)\right]=[\pi G(q, t)]^{-1 / 2} .
$$

Thus, within this approximation, using Eqs. (27) and (32) we get $P(q, t) \propto \sigma_{0}$ so that $\mu$ is independent of the nominal stress $\sigma_{0}$. Similarly, note that if we scale $E(\omega) \rightarrow \alpha E(\omega)$, then from Eqs. (27) and (32), $P(q, t) \propto 1 / \alpha$, so that $\mu$ depends only on the frequency variation of the complex elastic modulus, but not on its magnitude. For tires, the condition $\sigma_{0} \ll E(\omega=0)$ is usually satisfied. Consequently, on a dry road track one expects the same friction for wide and narrow 
tires, assuming that the rubber-road adhesional interaction and temperature effects are unimportant.

In order to take into account that $P(q, t) \rightarrow 1$ when $G(q, t) \rightarrow 0$, we use the interpolation formula

$$
P(q, t) \approx\left(1+[\pi G(q, t)]^{3 / 2}\right)^{-1 / 3} .
$$

Numerical evaluation of Eq. (26) shows that Eq. (33) is an accurate representation of $P(q, t)$ for all $q$ (or, equivalently, all $G$ ).

If we assume that the substrate surface is self-affine fractal on all length scales between an upper and lower cutoff, $\lambda_{0} \equiv 2 \pi / q_{0}$ and $\lambda_{1} \equiv 2 \pi / q_{1}$, we have $C(q)=0$ for $q<q_{0}$, while for $q>q_{0}$,

$$
C(q) \approx k\left(q / q_{0}\right)^{-2(H+1)},
$$

where $H=3-D_{f}$ (the fractal dimension $2<D_{f}<3$ ). If we define $\left\langle h^{2}\right\rangle=h_{0}^{2} / 2$, then Eq. (11) gives $k=\left(h_{0} / q_{0}\right)^{2} H / 2 \pi$. Using Eqs. (25) and (34) with $q=q_{0} \zeta$ gives

$$
\begin{aligned}
\mu(t)= & \frac{H}{4 \pi}\left(q_{0} h_{0}\right)^{2} \int_{1}^{q_{1} / q_{0}} d \zeta \zeta^{1-2 H} P(\zeta, t) \\
& \times \int d \phi \cos \phi \operatorname{Im} F(\mathbf{q}, t),
\end{aligned}
$$

where $P(\zeta, t)=A(\lambda) / A_{0}$ is given by Eq. (26) with

$$
G(q, t)=\frac{H}{16}\left(q_{0} h_{0}\right)^{2} \int_{1}^{q_{1} / q_{0}} d \zeta \zeta^{1-2 H} \int d \phi|F(\mathbf{q}, t)|^{2} .
$$

Note that since, to a good approximation, $P(q, t)$ $\sim[G(q, t)]^{-1 / 2}$, it follows that $P \sim 1 / q_{0} h_{0}$, and thus $\mu$ $\sim q_{0} h_{0}$.

\section{NUMERICAL RESULTS}

As an example, assume that $E$ is given by the model shown in Fig. 1(a). This model is, in fact, not a good description of real rubbers, since the transition with increasing frequency from the rubbery region to the glassy region is too abrupt, leading to a too narrow (and too high) $\mu_{k}(v)$ peak. Nevertheless, the model gives a qualitatively correct $E(\omega)$.

The model in Fig. 1(a) corresponds to the complex elastic modulus

$$
E(\omega)=\frac{E_{1}(1-i \omega \tau)}{1+a-i \omega \tau} .
$$

This function is shown in Fig. 1(b). Note that $E(\infty)=E_{1}$ and $E(0)=E_{1} /(1+a)$ so that $E(\infty) / E(0)=1+a$. Since typically $E(\infty) / E(0) \approx 1000$ we take $a=1000$ in all numerical calculations presented below. We assume $E_{1} \gg \sigma_{0}$, in which case $\mu(t)$ is independent of $E_{1}$ and $\sigma_{0}$. Note that

$$
E(t)=\int d \omega e^{-i \omega t} E(\omega)=2 \pi E_{1}\left[\delta(t)-\frac{a}{\tau} \theta(t) \mathrm{e}^{-(1+a) t / \tau}\right]
$$

so that

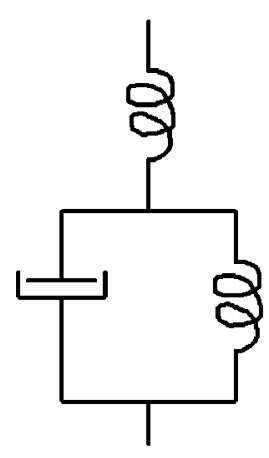

(a)

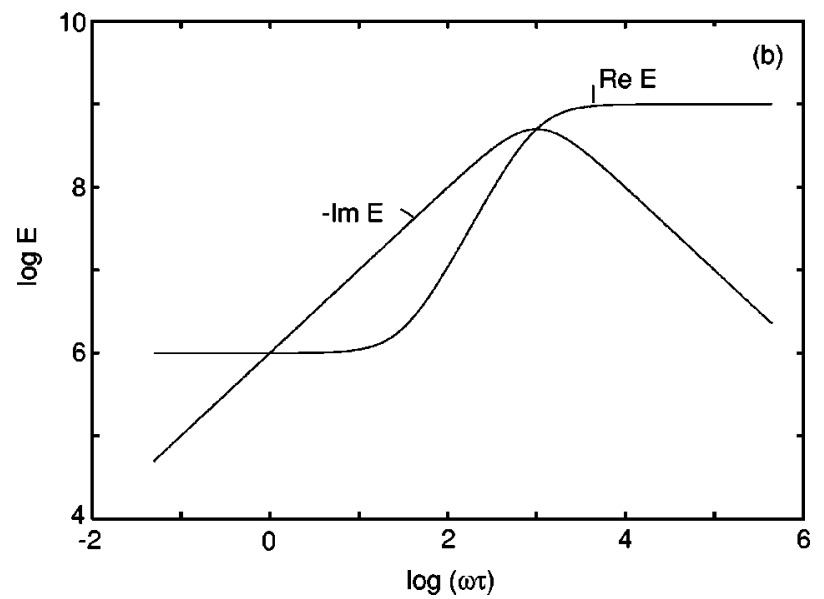

FIG. 1. (a) Rheological model corresponding to Eq. (37). (b) The complex elastic modulus $E(\omega)$ as a function of frequency. The logarithm has 10 as the basis. $E_{1}=10^{9} \mathrm{~N} / \mathrm{m}^{2}$ and $a=1000$.

$$
\begin{aligned}
& \frac{1}{2 \pi} \int d t^{\prime} E\left(t-t^{\prime}\right) e^{-i q_{x}\left[x(t)-x\left(t^{\prime}\right)\right]} \\
& \quad=E_{1}\left[1-\frac{a}{\tau} \int_{-\infty}^{t} d t^{\prime} e^{-(1+a)\left(t-t^{\prime}\right) / \tau} e^{-i q_{x}\left[x(t)-x\left(t^{\prime}\right)\right]}\right] .
\end{aligned}
$$

In what follows we consider two different histories for $x(t)$ : (i) Sliding velocity step-change:

$$
\begin{array}{ll}
x(t)=v_{0} t & \text { for } \quad t<0, \\
x(t)=v_{1} t \quad \text { for } & t>0 .
\end{array}
$$

Substituting this in Eq. (30) and using Eqs. (38b) gives

$$
\begin{aligned}
F= & -\frac{a}{1+a} E_{1}\left(1+\frac{1}{a}-\frac{\theta(-t)}{1+i q_{x} v_{0}}\right. \\
& \left.-\theta(t)\left[\frac{e^{-t-i q_{x} v_{1} t}}{1+i q_{x} v_{0}}+\frac{1-e^{-t-i q_{x} v_{1} t}}{1+i q_{x} v_{1}}\right]\right),
\end{aligned}
$$

where $t$ is measured in units of $\tau^{*}=\tau /(1+a), v$ in units of $1 /\left(q_{0} \tau^{*}\right)$, and $q$ is measured in units of $q_{0}$. (ii) Stop and start: 


$$
\begin{gathered}
x(t)=v_{0} t \quad \text { for } \quad t<0, \\
x(t)=0 \quad \text { for } \quad 0<t<T, \\
x(t)=v_{1}(t-T) \quad \text { for } t>T .
\end{gathered}
$$

Substituting this in Eq. (30) and using Eq. (38b) gives

$$
\begin{aligned}
F= & -\frac{a}{1+a} E_{1}\left(1+\frac{1}{a}-\frac{\theta(-t)}{1+i q_{x} v_{0}}\right. \\
& -\theta(t) \theta(T-t)\left[\frac{e^{-t}}{1+i q_{x} v_{0}}+1-e^{-t}\right] \\
& -\theta(t-T)\left[\frac{e^{-t-i q_{x} v_{1}(t-T)}}{1+i q_{x} v_{0}}+e^{-(t-T)-i q_{x} v_{1}(t-T)}\right. \\
& \left.\left.-e^{-t-i q_{x} v_{1}(t-T)}+\frac{1-e^{-(t-T)-i q_{x} v_{1}(t-T)}}{1+i q_{x} v_{1}}\right]\right),
\end{aligned}
$$

where again $t$ is measured in units of $\tau^{*}$, wave vector $q$ in units of $q_{0}$, and velocity $v$ in units of $1 /\left(q_{0} \tau^{*}\right)$.

Note that $\mu(t)$ depends on $H$ and $q_{0} h_{0}$. In what follows we use $H=0.8$ and $q_{0} h_{0}=1$. Since $\mu \sim q_{0} h_{0}$, the friction coefficient for other $q_{0} h_{0}$ can be obtained from direct scaling.

Figure 2(a) shows the kinetic friction coefficient as a function of the logarithm (with 10 as the basis) of the sliding velocity. The maximal friction occurs at a velocity $v_{c}$ where the substrate surface asperities give rise to fluctuating forces acting on the rubber with frequencies occurring in the transition region between the rubbery region and the glassy region in the mechanical response of the solid. For real rubber the peak maximum of $\mu_{k}(v)$ is smaller than in Fig. 2(a), and $\mu_{k}$ does not decrease towards zero for "large" and "small" velocities as in Fig. 2(a), but levels off at $\mu_{k} \approx 0.2-0.4$. This is, at least in part, a result of the fact that for real rubber $\operatorname{Im} E(\omega)$ does not decrease as rapidly towards zero as it does for the model elastic modulus (37).

Figure 2(b) shows the logarithm of the relative area of contact $P=A(\zeta) / A(1)$ [where $A(1)$ is the nominal contact area] as a function of the logarithm of the sliding velocity. $P$ is shown for two different magnifications, $\zeta=3$ and $\zeta=100$. Note that $P$ decreases from a constant value in the rubbery region to another much smaller value in the glassy region. The drop in magnitude corresponds to the factor $E(\infty) / E(0)=1+a=1001$. The physical reason for this is clear: at low sliding velocities the perturbing frequencies acting on the rubber surface from the surface asperities occur in the rubbery region where $E(\omega) \approx E(0)$ so the rubber is very soft. At high sliding velocities the perturbing frequencies are very high and correspond to the glassy region where $E(\omega)$ $\approx E(\infty)=(1+a) E(0)$. Since the area of real contact is roughly proportional to $1 / E$ the observed results follow.

Figure 3 shows (i) the friction coefficient $\mu(t)$, and (ii) the relative area of contact $P=A(\zeta) / A(1)$ as a function of time when the sliding velocity changes abruptly from $v_{0}$ $=10^{-4}$ to $v_{1}=10^{-3}$ at $t=0$. The relative area of contact is shown at two different magnifications, $\zeta=3$ and $\zeta=100$. The time and the sliding velocity are measured in natural units,

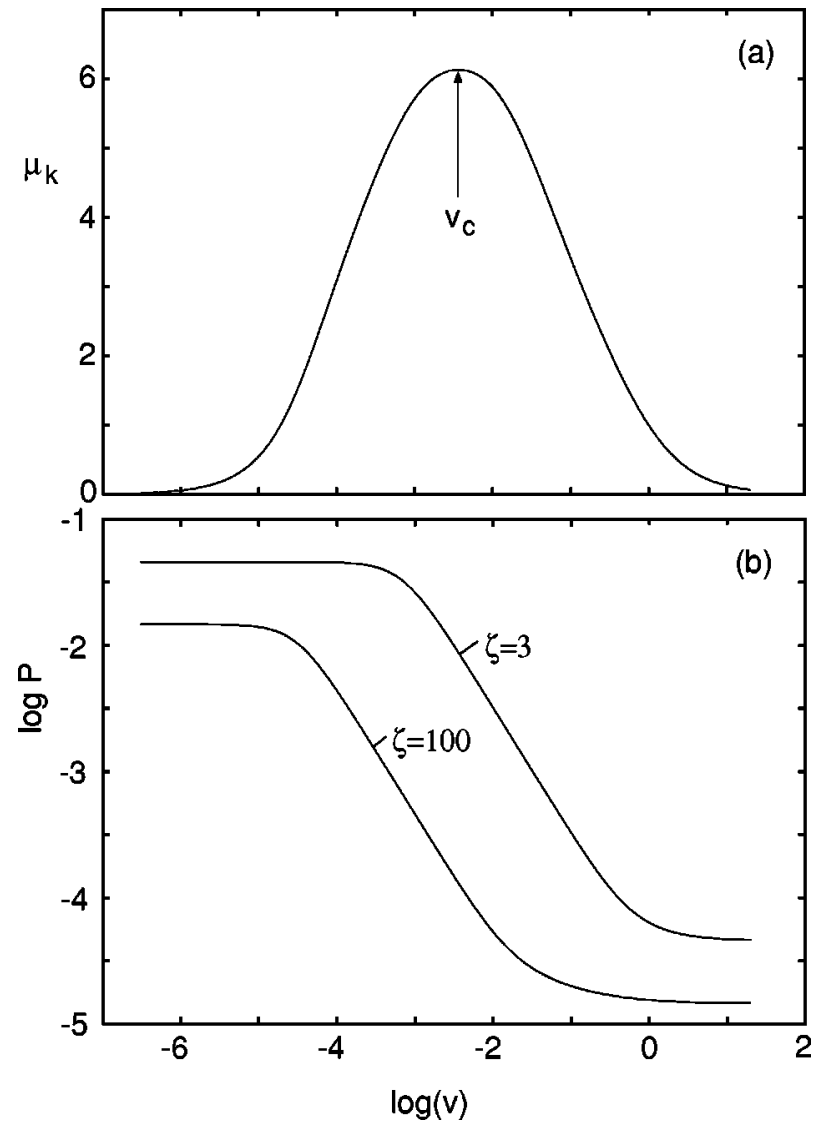

FIG. 2. (a) Kinetic friction coefficient $\mu_{k}$, and (b) relative area of contact $P=A(\zeta) / A(1)$, as a function of the logarithm (with 10 as the basis) of the sliding velocity. The relative area of contact is shown at two different magnifications, $\zeta=3$ and $\zeta=100$. The sliding velocity is in natural units $1 /\left(q_{0} \tau^{*}\right)$ (see text). $H=0.8, q_{0} h_{0}$ $=1, a \sigma_{0} / E_{1}=0.03, a=1000$, and $\zeta_{\max }=100$.

$\tau^{*}$ and $1 /\left(q_{0} \tau^{*}\right)$, respectively. Note that $v_{0}$ and $v_{1}$ are both to the left of the peak maximum in Fig. 2 (i.e., $v_{0}<v_{c}$ and $\left.v_{1}<v_{c}\right)$; in this case the friction coefficient changes monotonically between the steady-state values $\mu_{k}\left(v_{0}\right)$ and $\mu_{k}\left(v_{1}\right)$. Similarly, for $t>0$ the relative contact area $P$ decreases monotonically towards the steady-state value taken at the velocity $v_{1}$. A similar effect is observed when the sliding velocity changes abruptly from $v_{0}>v_{c}$ to $v_{1}>v_{c}$, both located to the right of the peak maximum in Fig. 2(a). This is illustrated in Fig. 4, which shows the same as Fig. 3, but now when the velocity changes (at $t=0$ ) from $v_{0}=0.01>v_{c}$ to $v_{1}=0.1$. The situation is drastically different, however, if the velocity changes from $v_{0}<v_{c}$ to $v_{1}>v_{c}$ (or vice versa). This is shown in Fig. 5 for the case when $v_{0}=10^{-4}$ and $v_{1}$ $=0.1$. In this case the sliding friction exhibits a characteristic peak (stiction spike), with the height $\Delta \mu \approx \mu_{k}\left(v_{c}\right)-\mu_{k}\left(v_{0}\right)$ and the width $\Gamma \sim \tau^{*}$. This result is easy to understand: Assume that the sliding velocity changes abruptly from $v_{0}$ $\left\langle v_{c}\right.$ to $v_{1}>v_{c}$. Thus, the velocity of the solid at the interface will, during a very short time period, increase from $v_{0}$ to $v_{1}$. If we assume that the frictional shear stress is given (approximately) by the instantaneous value, $\mu(t) \approx \mu[\dot{x}(t)]$, then it follows that there must be a friction spike of height 


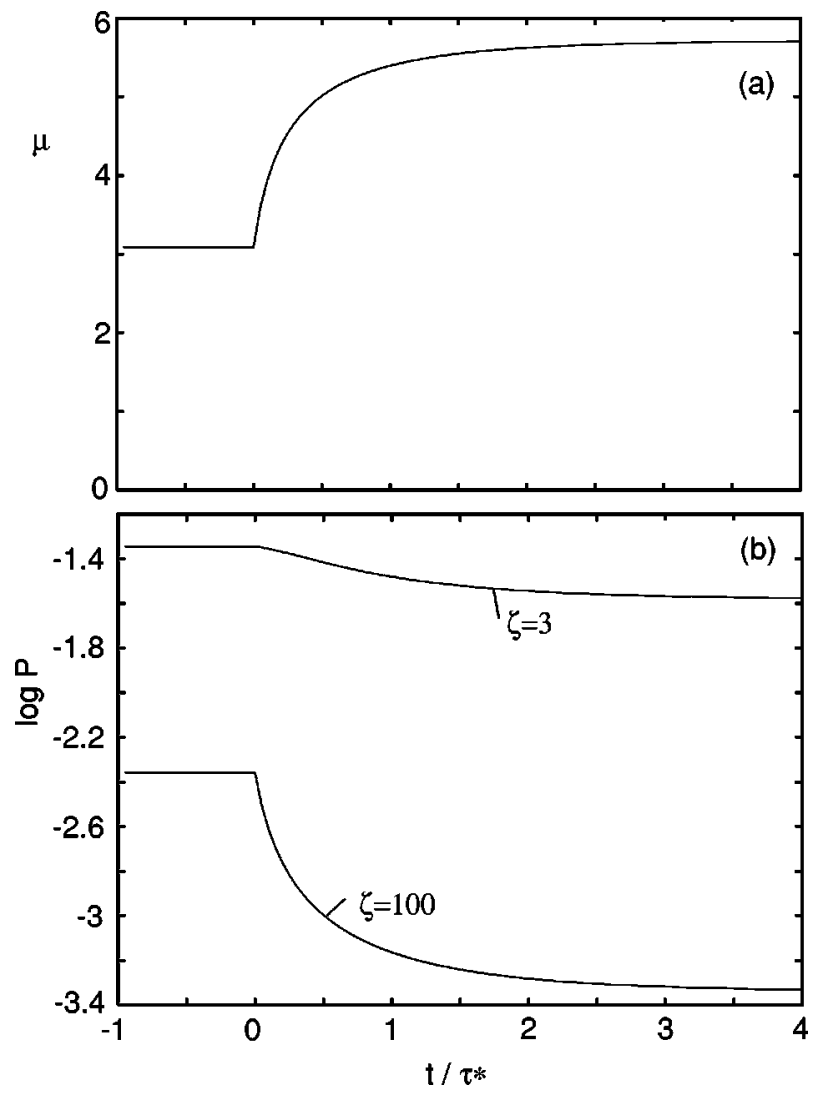

FIG. 3. (a) Friction coefficient $\mu(t)$, and (b) relative area of contact $P=A(\zeta) / A(1)$, as a function of time when the sliding velocity changes abruptly from $10^{-4}$ to $10^{-3}$ at $t=0$. The relative area of contact is shown at two different magnifications, $\zeta=3$ and $\zeta=100$. The time and the sliding velocity are in natural units, $\tau^{*}$ and $1 /\left(q_{0} \tau^{*}\right)$, respectively (see text). $H=0.8, q_{0} h_{0}=1, a \sigma_{0} / E_{1}$ $=0.03, a=1000$, and $\zeta_{\max }=100$.

$\Delta \mu=\mu\left(v_{c}\right)-\mu\left(v_{0}\right)$ as indeed observed. This argument also predicts that no friction spike should occur if both $v_{0}$ and $v_{1}$ are below or above $v_{c}$, again in agreement with the calculations (see Figs. 3 and 4).

Figures 6 and 7 show the results of two stop-start calculations. Figure 6 shows a case where the sliding velocity is abruptly changed from $v_{0}=10^{-3}<v_{c}$ to zero, $t=0$, and then increased back to $v_{0}$ at $t=T=5$. Note that $\mu(t)$ decreases continuously during "stop" (relaxation), and then increases monotonically back to $\mu_{k}\left(v_{0}\right)$ for $t>5$. Similarly, the area of contact increases monotonically during "stop," and then at the onset of sliding decreases back towards its original (for $t<0$ ) value. It is interesting to note that the area of contact at the magnification $\zeta=100$ initially increases exponentially with time as $\sim \exp \left(t / \tau^{*}\right)$ giving a straight line in the $t$ $-\log P$ diagram with the slope $1 / \log (10)$. This result is easy to prove analytically: First note that when $v=v_{0}$ for $t<0$ and $v=v_{1}=0$ for $t>0$, from Eq. (39) we get

$$
F=\frac{1}{a}-\frac{i q_{x} v_{0}}{1+i q_{x} v_{0}}\left[1-\left(1-e^{-t}\right) \theta(t)\right] .
$$

Since in the present case $1 / a=10^{-3}$ while $q v_{0}>0.1$ we can neglect the $1 / a$ term in Eq. (41). Substituting $F(\mathbf{q}, t)$ in Eq.

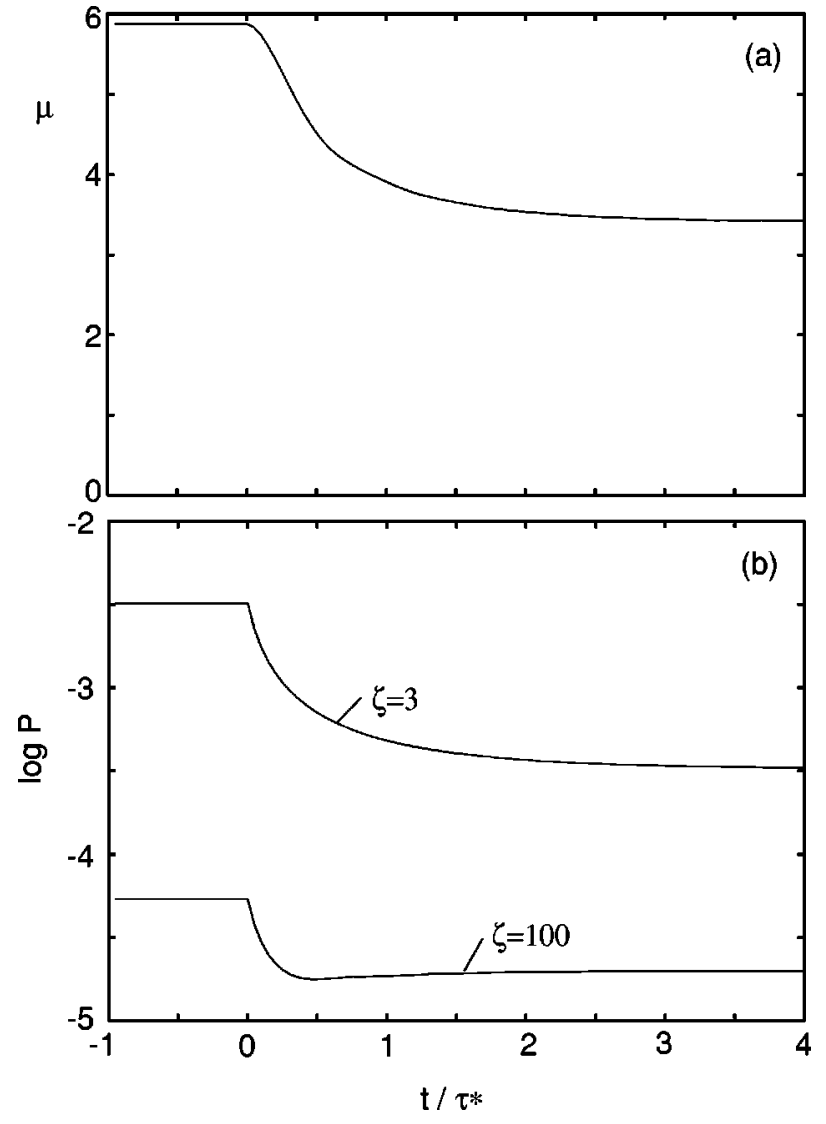

FIG. 4. (a) Friction coefficient $\mu(t)$, and (b) relative area of contact $P=A(\zeta) / A(1)$, as a function of time when the sliding velocity changes abruptly from 0.01 to 0.1 at $t=0$. The relative area of contact is shown at two different magnifications, $\zeta=3$ and $\zeta$ $=100$. The time and the sliding velocity are in natural units, $\tau^{*}$ and $1 /\left(q_{0} \tau^{*}\right)$, respectively (see text). $H=0.8, q_{0} h_{0}=1, \quad a \sigma_{0} / E_{1}$ $=0.03, a=1000$, and $\zeta_{\max }=100$.

(36) gives $G(q, t)=G_{0}(q)$ for $t<0$ and $G(q, t)$ $=G_{0}(q) \exp (-2 t)$ for $t>0$. As long as the area of real contact is small compared to the nominal contact area, we have [see Eq. (32) $] P \approx[\pi G(q, t)]^{-1 / 2}$ so that the area of contact $A(t)=A(0) \exp (t)$, or in real time units, $A(0) \exp \left(t / \tau^{*}\right)$, for $t>0$.

Figure 7 shows a case where the sliding velocity is abruptly changed from $v_{0}=0.1>v_{c}$ to zero (at $t=0$ ) and then switched back to $v_{0}$ at $t=T=5$. Note that the friction coefficient again decreases monotonically during "stop," but exhibits a stiction spike when $v$ is switched back to $v_{0}$. This is in sharp contrast to the case when $v_{0}<v_{c}$ (Fig. 6), and the origin of this difference is the same as presented before in the context of Fig. 5. Figure 7(b) shows that the area of contact increases monotonically during "stop" and then at the onset of sliding decreases back to its original (for $t<0$ ) value. In this case the contact area for both $\zeta=3$ and 100 initially increases exponentially with time as $\sim \exp \left(t / \tau^{*}\right)$ giving a straight line in the $t-\log P$ diagram with the slope $1 / \log (10)$. This result can be explained in the same way as in the context of Fig. 6.

Figure 8 shows the results of several stop-start calculations with $T=0.1,0.3,0.5,1,2,3,5$, and 10 . An analysis of 

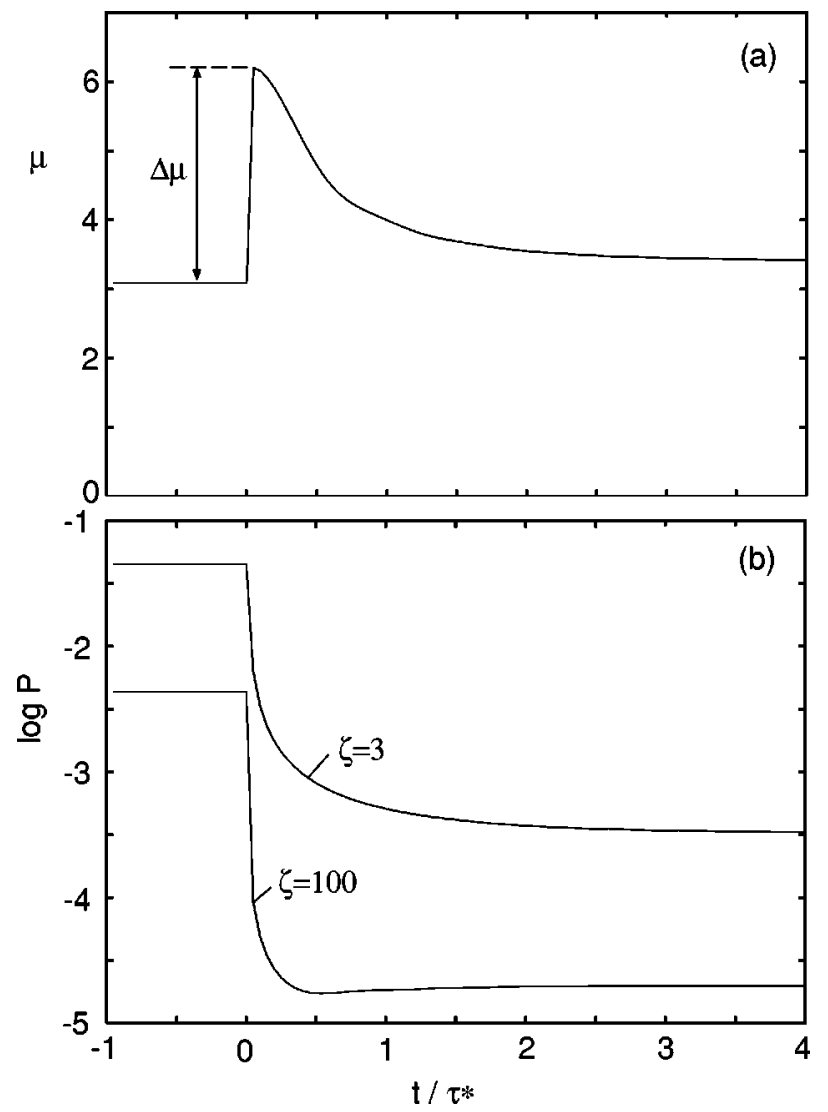

FIG. 5. (a) Friction coefficient $\mu(t)$, and (b) relative area of contact $P=A(\zeta) / A(1)$, as a function of time when the sliding velocity changes abruptly from $10^{-4}$ to 0.1 at $t=0$. The relative area of contact is shown at two different magnifications, $\zeta=3$ and $\zeta$ $=100$. The time and the sliding velocity are in natural units, $\tau^{*}$ and $1 /\left(q_{0} \tau^{*}\right)$, respectively (see text). $H=0.8, \quad q_{0} h_{0}=1, a \sigma_{0} / E_{1}$ $=0.03, a=1000$, and $\zeta_{\max }=100$.

the height $\Delta \mu$ of the stiction spikes shows that $\Delta \mu(T)$ increases logarithmically with stop time $T$ [see Fig. 9(a)] as long as the height of the stiction spike is much smaller than the limiting (saturation) level, $\Delta \mu(\infty) \approx 2.8$, observed for large stopping time. Thus the results in Fig. 8 for $T<3$ are well described by

$$
\Delta \mu=\mu_{\max }-\mu_{k}\left(v_{0}\right) \approx(\Delta \mu)_{0} \ln \left(1+\frac{T}{3 \tau^{*}}\right),
$$

where $(\Delta \mu)_{0} \approx 4$. This curve is given by the solid line in Fig. 9(a) while the circles are the data from Fig. 8. This type of logarithmic time dependence of $\Delta \mu$ on $T$ is often observed in experiments, ${ }^{22}$ but the origin of the effect for nonrubber materials is likely to be different from the present case (see Sec. IV). One contribution to the increase in the height $\Delta \mu(T)$ with increasing stopping time $T$ results from a corresponding increase in the contact area $\Delta A(T)$. If the average (over the contact area) shear stress $\sigma$ that is needed to start sliding would be independent of the stopping time, then one expects $\Delta \mu(T) \sim \Delta A(T)$. This formula is often assumed to hold, but Fig. 9(b) shows that this relation does not hold accurately in the present case; thus, $\sigma$ also will depend on the stopping

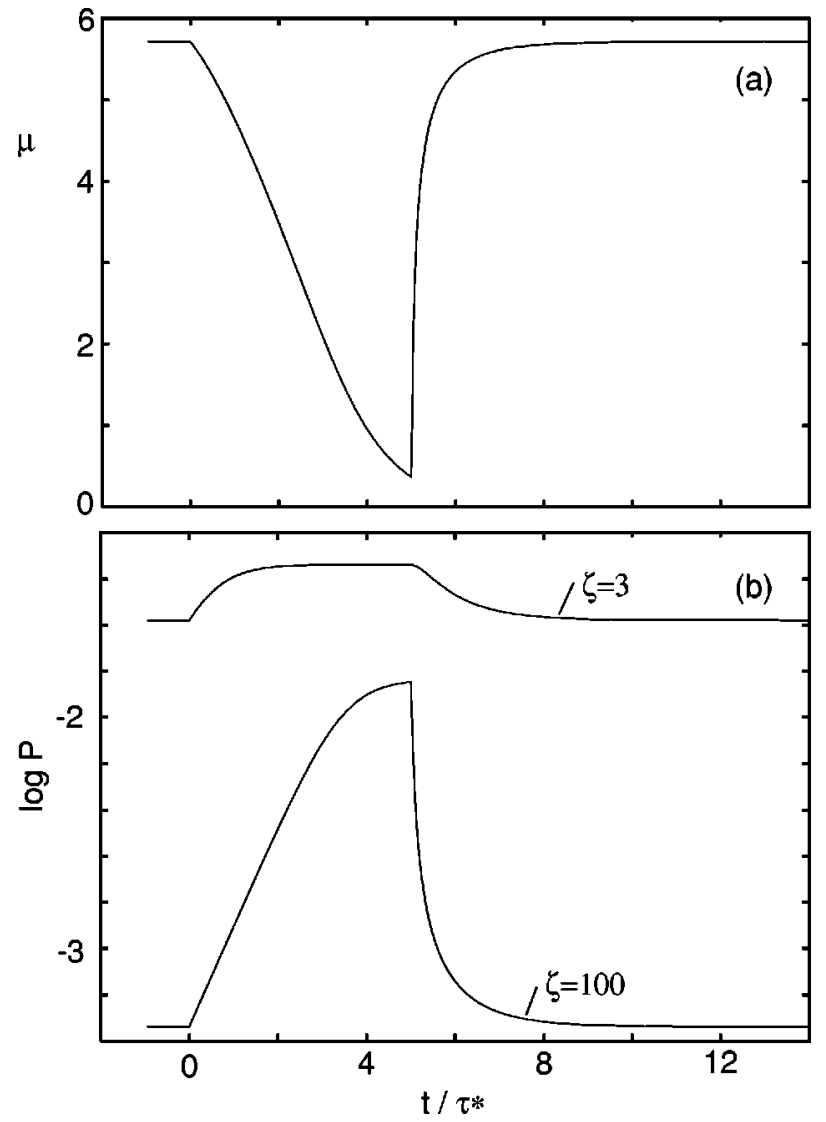

FIG. 6. (a) Friction coefficient $\mu(t)$, and (b) relative area of contact $P=A(\zeta) / A(1)$, as a function of time when the sliding velocity changes abruptly, reducing from $v_{0}=10^{-3}$ to 0 at $t=0$ and then returning to $v_{0}$ at $t=5$. The relative area of contact is shown at two different magnifications, $\zeta=3$ and $\zeta=100$. The time and the sliding velocity are in natural units, $\tau^{*}$ and $1 /\left(q_{0} \tau^{*}\right)$, respectively (see text). $H=0.8, q_{0} h_{0}=1, a \sigma_{0} / E_{1}=0.03, a=1000$, and $\zeta_{\max }=100$.

time. Figure 9(b) shows $\Delta \mu$ as a function of $\Delta A(T) / A_{0}$ for $\zeta=3$, as obtained from Fig. 8.

\section{DISCUSSION}

The theory developed above can be used to estimate the friction coefficient for nonsteady sliding of rubber on a rough, hard substrate. The input for the calculation, namely the complex elastic modulus $E(\omega)$ and information about the substrate roughness [spectral function $C(q)$ ] can be obtained directly from relatively simple experiments.

The theory relates the friction force to the coordinate $x(t)$ of the bottom surface of the rubber block. However, the external driving force does not act directly on the bottom surface of the block, but usually on the top surface or at some other distant area. When studying the motion of the rubber block it is, in general, necessary to include the elastic deformation of the rubber between the bottom surface of the block and the area where the external forces act. Consider, for example, a rectangular elastic block. Assume that the upper surface of the block is "glued" to a thin rigid sheet as indi- 


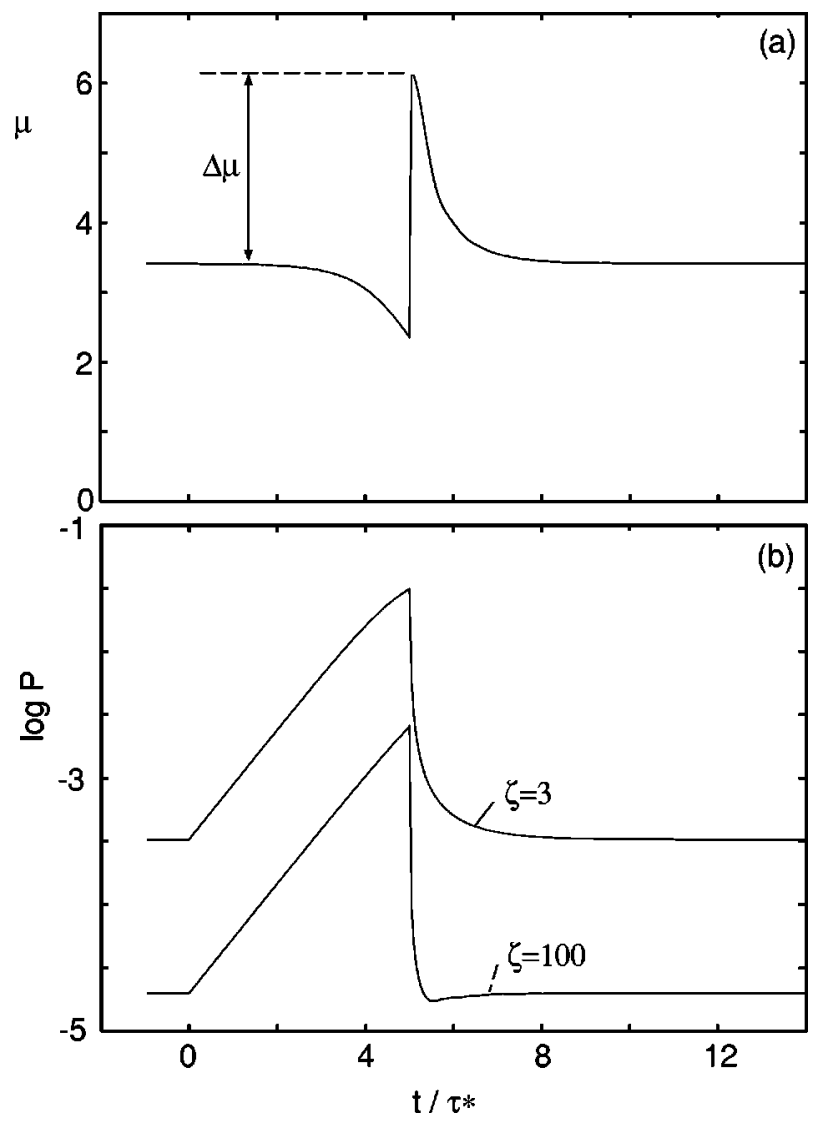

FIG. 7. (a) Friction coefficient $\mu(t)$, and (b) relative area of contact $P=A(\zeta) / A(1)$, as a function of time when the sliding velocity changes abruptly, reducing from $v_{0}=0.1$ to 0 at $t=0$ and then returning to $v_{0}$ at $t=5$. The relative area of contact is shown at two different magnifications, $\zeta=3$ and $\zeta=100$. The time and the sliding velocity are in natural units, $\tau^{*}$ and $1 /\left(q_{0} \tau^{*}\right)$, respectively (see text). $H=0.8, \quad q_{0} h_{0}=1, a \sigma_{0} / E_{1}=0.03, a=1000$, and $\zeta_{\max }=100$.

cated in Fig. 10. If a parallel force is applied to the rigid sheet, either directly, or, as is typical in many applications, via an external spring (spring constant $k_{s}$ ), then the block will deform elastically as indicated in the figure. This elastic coupling between the sliding interface, where the friction force is generated, and the point or area where the driving force acts must be taken into account when studying sliding dynamics of the block, and is particularly important for materials with a low elastic modulus such as rubber. We will study this problem in another publication.

Let us point out that in addition to the contribution to rubber friction from the internal friction of the rubber studied above, there will in general be other contributions arising from pinning effects at the interface. Thus, for a clean rubber surface (if that ever exists) in contact with a hard substrate, the rubber molecules at the interface will rearrange themselves to bind as strongly as possible to the substrate surface. Because of the lateral corrugation of the substrate potential this will in general give rise to an energy barrier towards sliding. If the rubber is in contact with another polymer surface, e.g., rubber in contact with rubber, chain interdiffusion may also occur at the interface which will give a contribution
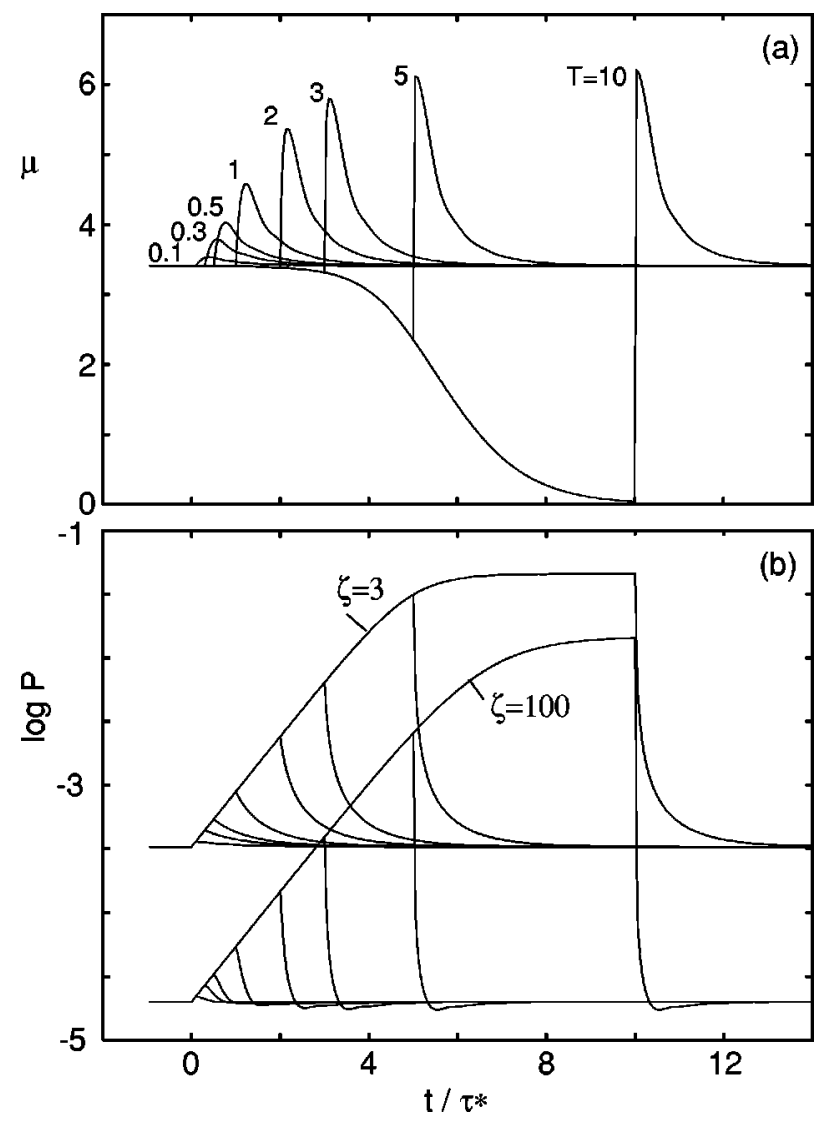

FIG. 8. (a) Friction coefficient $\mu(t)$, and (b) relative area of contact $P=A(\zeta) / A(1)$, as a function of time when the sliding velocity changes abruptly reducing from $v_{0}=0.1$ to 0 at $t=0$ and then returning to $v_{0}$ at $t=T=0.1,0.3,0.5,1,2,3,5$, and 10 . The relative area of contact is shown at two different magnifications, $\zeta=3$ and $\zeta=100$. The time and the sliding velocity are in natural units, $\tau^{*}$ and $1 /\left(q_{0} \tau^{*}\right)$, respectively (see text). $H=0.8, q_{0} h_{0}=1, a \sigma_{0} / E_{1}$ $=0.03, a=1000$, and $\zeta_{\max }=100$.

to both the static and kinetic friction forces. Finally, since most real surfaces are contaminated with a few monolayers of physisorbed organic molecules, the contamination layer will also contribute the friction force as discussed in detail in many recent publications. ${ }^{21,7}$ All these additional contributions to the rubber friction give a contribution to the friction coefficient which is typically of order $\mu \sim 0.2$.

Let us comment on the concept of the static friction force. If there would be no interfacial pinning processes of the type described above, then, strictly speaking, the static friction force would vanish. However, assume that the $E(\omega)$ function has the form shown in Fig. 11(a), giving rise to a kinetic friction coefficient $\mu_{k}(v)$ of the form shown in Fig. 11(b). Assume now that we start to pull the block with some speed $v_{1}$ indicated in Fig. 11(b). In this case we would observe a stiction spike (or static friction coefficient) of height $\sim \mu_{k}\left(v_{a}\right)$, see Fig. 11(c). Thus, if there are very lowfrequency (long-time) relaxation processes in the solid [corresponding to the low-frequency peak in Fig. 11(a)], they may show up as a static friction force under most normal sliding friction experiments. However, if the sliding velocity is extremely small $\left(v<v_{a}\right)$ there would be no stiction spike, 

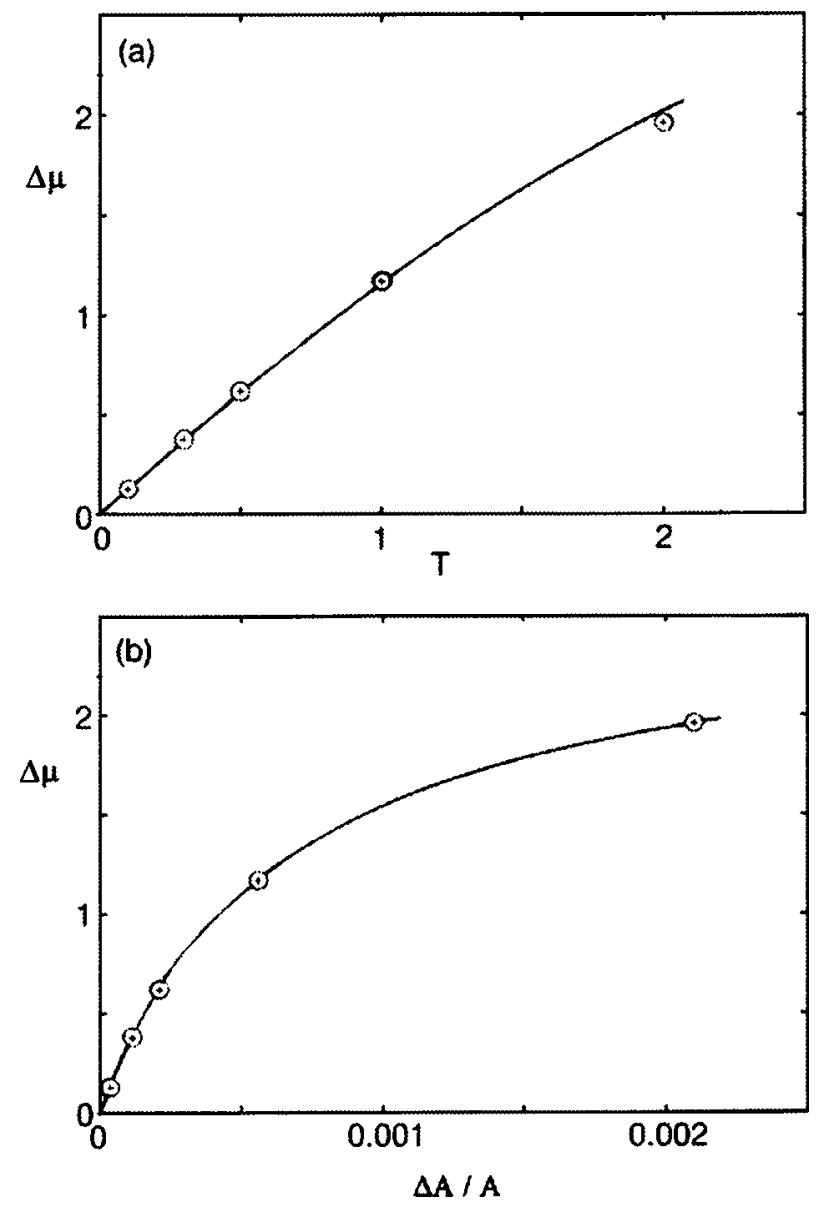

FIG. 9. (a) The stiction $\Delta \mu(T)$ as a function of the stopping time $T$ (from Fig. 8). The solid line is given by Eq. (42). (b) The stiction $\Delta \mu(T)$ as a function of the increase of the relative area of contact $\Delta A(\zeta) / A(1)$ (for $\zeta=3$ ) (from Fig. 8). The time $T$ is in units of $\tau^{*} . H=0.8, q_{0} h_{0}=1, a \sigma_{0} / E_{1}=0.03, a=1000$, and $\zeta_{\max }=100$.

which could be interpreted as the absence of a static friction force. Thus, there is no single value of the static friction coefficient - it depends upon the initial dwell time and rate of starting (which, according to Fig. 10, also depends on the rubber modulus). ${ }^{19}$

In many sliding friction experiments it has been observed that the height $\Delta \mu(T)$ of the stiction spike in stop-start experiments increases logarithmically with the stopping time. A similar effect was observed above in our model calculations

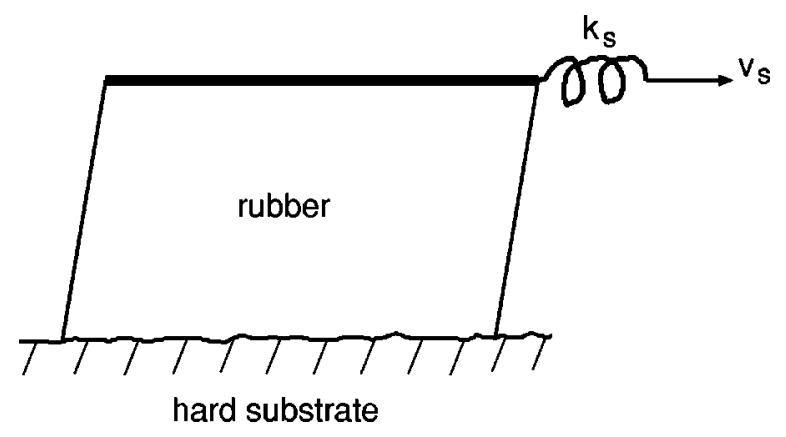

FIG. 10. A rubber block on a rough substrate.
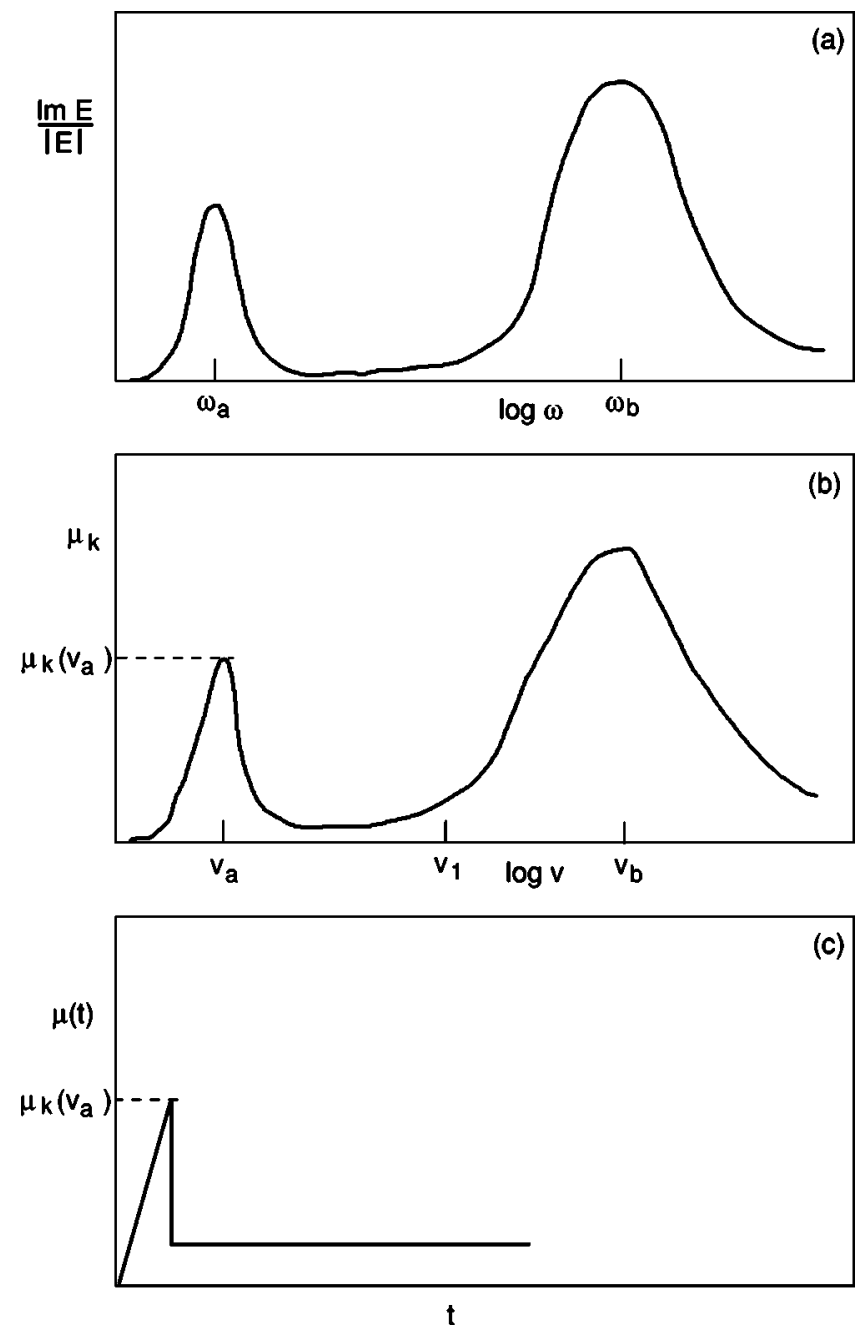

FIG. 11. (a) The "loss spectra" $\operatorname{Im} E(\omega) /|E(\omega)|$ as a function of frequency $\omega$. (b) The kinetic friction coefficient. (c) The timedependent friction coefficient (defined as the time-dependent friction force divided by the load).

for rubber. However, we believe that the origin of the $\Delta \mu$ $\sim \ln T$ relation observed for nonrubber materials ${ }^{22}$ (e.g., stone, paper, or plastic) has a different origin, unrelated to the internal friction of these materials: During steady sliding at low sliding velocities a wide distribution of tangential stresses will occur at the sliding interface. Thus, surface asperities $^{23}$ or stress domains ${ }^{24}$ in the contact area form a wide distribution of elastically deformed states which during "stop" will slowly relax (by thermal excitation over the barriers) towards the equilibrium (unstressed) state. This process can be shown ${ }^{24}$ to give a $\Delta \mu(T)$ which depends logarithmically on the stopping time $T$. The same process gives rise to a logarithmic dependence of the kinetic friction coefficient $\mu_{k}$ on the sliding velocity (for low sliding velocities), again in agreement with experiments. This latter effect is not observed for rubber unless the rubber is probed well into the glassy region (which requires "low" enough temperature compared to the rubber glass transition temperature), where, in fact, rubber behaves like most other solid materials, and where the friction no longer is dominated by the internal 
friction, but rather by interfacial processes as described above.

\section{SUMMARY AND CONCLUSION}

There is at present a strong drive by tire companies to design new rubber compounds with lower rolling resistance, higher sliding friction, and reduced wear. At present these attempts are mainly based on a few empirical rules and on very costly trial-and-error procedures. We believe that a fundamental understanding of rubber friction and wear may help in the design of new rubber compounds for tires and other rubber applications, e.g., wiper blades or products for the cosmetic industry.

In this paper we have presented a general theory of the hysteretic contribution to rubber friction for nonstationary sliding. The theory has been developed for rubber sliding on a hard self-affine fractal surface, e.g., a tire on a road surface. Numerical results were presented for a simple rheological model of the complex elastic modulus $E(\omega)$, but the theory has been developed for arbitrary $E(\omega)$, e.g., the experimentally measured $E(\omega)$ can be directly used as an input. The theory relates the friction force to the coordinate of the bottom surface of the rubber block. In most practical applications the external driving force acts some distance away from the sliding interface, and it is then necessary to include in the equations of motion the elastic coupling between the bottom surface of the rubber block and the area where the external driving force acts.

\section{ACKNOWLEDGMENTS}

This work has been supported by a research and development grant from Pirelli Pneumatici. We thank O. Albohr, G. Heinrich, M. Klüppel, U. Kuhlmann, G. Luengo, and V. Peveri for useful discussions. B.P. also thanks BMBF for a grant related to the German-Israeli Project Cooperation, "Novel Tribological Strategies from the Nano to Meso-Scales."
${ }^{1}$ D.F. Moore, The Friction and Lubrication of Elastomer (Pergamon Press, Oxford, 1972); M. Barquins, Mater. Sci. Eng. 73, 45 (1985); A.D. Roberts, Rubber Chem. Technol. 65, 673 (1992).

${ }^{2} \mathrm{G}$. Luengo (private communication).

${ }^{3}$ K.A. Grosch, Proc. R. Soc. London, Ser. A 274, 21 (1963); see also, A.D. Roberts, Rubber Chem. Technol. 65, 3 (1992); S.P. Arnold, A.D. Roberts, and A.D. Taylor, J. Nat. Rubber Res. 2, 1 (1987); M. Barquins and A.D. Roberts, J. Phys. D 19, 547 (1986).

${ }^{4}$ M. Klüppel and G. Heinrich, Rubber Chem. Technol. 73, 578 (2000).

${ }^{5}$ B.N.J. Persson, J. Chem. Phys. 115, 3840 (2001).

${ }^{6}$ B.N.J. Persson, Surf. Sci. 401, 445 (1998).

${ }^{7}$ B.N.J. Persson, Sliding Friction: Physical Principles and Applications, 2nd ed. (Springer, Heidelberg, 2000).

${ }^{8}$ B.N.J. Persson and E. Tosatti, J. Chem. Phys. 112, 2021 (2000).

${ }^{9}$ A. Schallamach, Wear 6, 375 (1963); Y.B. Chernyak and A.I. Leonov, ibid. 108, 105 (1986).

${ }^{10}$ G. Heinrich, Kautsch. Gummi Kunstst. 45, 173 (1992); Rubber Chem. Technol. 70, 1 (1997).

${ }^{11}$ J. Feder, Fractals (Plenum Press, New York, 1988).

${ }^{12}$ E. Bouchaud, J. Phys.: Condens. Matter 9, 4319 (1997).
${ }^{13}$ J.F. Archard, Proc. R. Soc. London, Ser. A 243, 190 (1957).

${ }^{14}$ J.A. Greenwood, in Fundamentals of Friction, Macroscopic and Microscopic Processes, edited by I.L. Singer and H.M. Pollack (Kluwer, Dordrecht, 1992).

${ }^{15}$ S. Roux, J. Schmittbuhl, J.P. Vilotte, and A. Hansen, Europhys. Lett. 23, 277 (1993).

${ }^{16}$ A. Majumdar and B. Bhushan, J. Tribol. 113, 1 (1991).

${ }^{17}$ J.A. Greenwood and J.B.P. Williamson, Proc. R. Soc. London, Ser. A 295, 300 (1966).

${ }^{18}$ O. Ronsin and K.L. Coeyrehourcq, Proc. R. Soc. London, Ser. A 457, 1277 (2001).

${ }^{19}$ A.D. Roberts and A.G. Thomas, Rubber Chem. Technol. 50, 266 (1977).

${ }^{20}$ B.N.J. Persson, Phys. Rev. B 63, 104101 (2001).

${ }^{21}$ B.N.J. Persson, J. Chem. Phys. 113, 5477 (2000); M.H. Müser, L. Wenninger, and M.O. Robbins, Phys. Rev. Lett. 86, 1295 (2001); J. Gao, W.D. Luedtke, and U. Landman, Tribol. Lett. 9, 3 (2000).

${ }^{22}$ P. Berthoud, T. Baumberger, C. G'Sell, and J.M. Hiver, Phys. Rev. B 59, 14313 (1999).

${ }^{23}$ C. Caroli and P. Nozieres, in Physics of Sliding Friction, edited by B.N.J. Persson and E. Tosatti (Kluwer, Dordrecht, 1996).

${ }^{24}$ B.N.J. Persson, Phys. Rev. B 51, 13568 (1995). 